\title{
Clinical characteristics and outcome of SARS-CoV-2-infected patients with haematological diseases: a retrospective case study in four hospitals in Italy, Spain and the Netherlands
}

\author{
Jaap van Doesum $\mathbb{1}^{1} \cdot$ Anabelle Chinea $\mathbb{E}^{2} \cdot$ Maria Pagliaro $^{3} \cdot$ Maria Cristina Pasquini ${ }^{4} \cdot$ Tom van Meerten ${ }^{1} \cdot$ \\ Martijn Bakker ${ }^{1} \cdot$ Emanuele Ammatuna $^{1}$
}

Received: 16 June 2020 / Revised: 23 June 2020 / Accepted: 30 June 2020 / Published online: 8 July 2020

(c) Springer Nature Limited 2020

\section{To the Editor:}

We have read with great interest the paper of $\mathrm{He}$ et al. published in April 2020 about haematological patients infected with SARS-COV-2 [1]. Although it was a small series with 13 patients, it gave a good insight into the course and outcome of the infection in this patient category. Interestingly, limited data about SARS-COV-2 in haematological patients have become available since this report, with just two other cohorts published [2, 3]. Because of the high mortality in this vulnerable group of patients, it is important to have more information about the course and outcome of this disease in larger patient series. We describe in this letter a cohort of 59 COVID-19 patients with an underlying haematological disease from three European countries.

This retrospective case study was performed in four hospitals across Europe: Spain (Hospital Ramón y Cajal, Madrid), Northern-Italy (ASST, Crema, and San Giovanni Bosco General Hospital, Turin) and the Netherlands (University Medical Center Groningen). Patients with COVID-19 infection and a concomitant haematological disease were identified between 10 February 2020 and 15 May 2020. COVID-19 diagnosis was confirmed

Jaap van Doesum

j.a.van.doesum@umcg.nl

1 Department of Hematology, University Medical Center Groningen, Groningen, The Netherlands

2 Department of Hematology, Hospital Ramón y Cajal, Madrid, Spain

3 Hematology Unit, San Giovanni Bosco General Hospital, Torino, Italy

4 Oncology Unit, ASST of Crema, Crema, Italy with a positive RT-PCR assay. Clinical data were retrospectively retrieved from the medical records, including clinical features, laboratory findings, imaging, treatment and outcome.

We identified 59 patients with a haematological disease and concomitant COVID-19 infection. Their mean age was 67 years (range 32-92) and 54\% were male. Thirty-three patients $(56 \%)$ had a lymphoid malignancy and 20 patients (34\%) suffered from a myeloid malignancy. A relative high incidence of patients (10\%) had an idiopathic thrombocytopenic purpura (Table 1).

Thirty-nine (66\%) patients were being treated for their underlying disease at the time of COVID-19 diagnosis (Table 1). The mean duration of symptoms before the diagnosis of COVID-19 was 5.8 days (range 0-34). Eightyeight percent of patients had a community-acquired infection and 54\% had metabolic comorbidity (e.g. hypertension, diabetes, obesity or cardiovascular events). The most common presenting symptoms were fever (93\%), dyspnoea (62\%), dry cough (47\%) and diarrhoea (29\%). Almost all patients (94\%) had CT imaging abnormalities characteristic of COVID-19. The most common radiologic findings were ground glass opacifications. Four patients $(7 \%)$ had a neutropenia at presentation and $23(40 \%)$ a lymphopenia. The different treatments given for COVID-19 and their outcome are shown in Table 2.

Seven patients with respiratory failure did not start mechanical ventilation due to the underlying advanced haematological disease. Five patients $(8.5 \%)$ developed a thrombotic event during follow-up, mostly pulmonary embolisms.

At last follow-up 20 patients (34\%) died due to COVID-19. The mortality rate for patients above 60 years was $45 \%$, and that for patients below 60 years was $11 \%$. There was no difference in survival between lymphoid and myeloid malignancies. In addition, we did not observe 
Table 1 Baseline demographic and clinical characteristics of patients infected by SARS-COV-2.

\begin{tabular}{|c|c|}
\hline Demographics & Patients $(N=59)$ \\
\hline Age, years (range) & $67(32-92)$ \\
\hline Male sex, $\%$ & 54 \\
\hline \multicolumn{2}{|l|}{ Patients' hospital } \\
\hline Hospital Ramón y Cajal, Madrid, $N$ & 29 \\
\hline ASST, Crema, $N$ & 14 \\
\hline University Medical Center, Groningen, $N$ & 8 \\
\hline San Giovanni Bosco General Hospital, Turin, $N$ & 8 \\
\hline \multicolumn{2}{|l|}{ Haematological disease } \\
\hline Lymphoid malignancies, $N(\%)$ & $33(56)$ \\
\hline Hodgkin lymphoma, $N(\%)$ & $2(3,4)$ \\
\hline Multiple myeloma, $N(\%)$ & $10(16,9)$ \\
\hline Chronic lymphatic leukaemia, $N(\%)$ & $5(8,5)$ \\
\hline Non-Hodgkin lymphoma, $N(\%)$ & $15(25,5)$ \\
\hline Acute lymphoblastic leukaemia, $N(\%)$ & $1(1,7)$ \\
\hline Myeloid malignancies, $N(\%)$ & $20(34)$ \\
\hline Acute myeloid leukaemia, $N(\%)$ & $6(10)$ \\
\hline Myelodysplastic syndrome, $N(\%)$ & $3(5,1)$ \\
\hline Myeloproliferative neoplasms, $N(\%)$ & $11(18,6)$ \\
\hline Benign haematological disease, $N(\%)$ & $6(10)$ \\
\hline Idiopathic thrombocytopenic purpura $N(\%)$ & $6(10)$ \\
\hline \multicolumn{2}{|l|}{ Last haematological treatment } \\
\hline Haematopoietic cell transplantation, $N(\%)$ & $2(3,4)$ \\
\hline Chemotherapy $<30$ days, $N(\%)$ & $23(39,0)$ \\
\hline Immunotherapy $<30$ days, $N(\%)$ & $29(49,1)$ \\
\hline Rituximab & $9(15,3)$ \\
\hline Steroids & $16(27,1)$ \\
\hline Daratumumab & $1(1,7)$ \\
\hline Tyrosine kinase inhibitor & $3(5,1)$ \\
\hline
\end{tabular}

any difference in survival between the different treatment strategies of COVID-19 infection.

To the best of our knowledge this is the second European series of patients with COVID-19 and a haematological disease [3]. The estimated 1-month overall survival is $71 \%$, which conforms to the survival rate of haematological patients published by Lee et al. and that of other series of patients with a malignancy [2-4]. It must be noted that like other case series the average age of our series is above 60 years and more than $50 \%$ of patients had metabolic comorbidities. In the series of Malard et al. there was an overrepresentation of patients with a multiple myeloma [2]. This could not be confirmed in our multinational cohort, although lymphoid malignancies seem to be more common. In our series $92 \%$ of the patients needed to be hospitalised, so our data is biased due to the fact that only patients with severe or critical illness were tested due to the limited availability of
Table 2 Treatment and outcome of COVID-19-infected patients with a haematological disease.

\begin{tabular}{ll}
\hline Hospitalisation & \\
$\quad$ Number of patients hospitalised, $N(\%)$ & $54(92)$ \\
Hospitalisation duration, days (range) & 12 days (1-61) \\
Oxygen therapy & $47(79)$ \\
Oxygen support needed, $N(\%)$ & $14(24)$ \\
Mechanical ventilation needed, $N(\%)$ & $8(14)$ \\
Non-invasive ventilation/continuous positive & \\
airway pressure, $N(\%)$ & $5(0-20)$ \\
Mean duration between COVID-19 diagnosis and \\
starting mechanical ventilation, days (range) \\
Medicine treatment \\
Steroids, $N(\%)$ & $29(48)$ \\
(Hydroxy)chloroquine, $N(\%)$ & $48(80)$ \\
Tocilizumab, $N(\%)$ & $7(12)$ \\
Azithromycin, $N(\%)$ & $28(47)$ \\
Lopinavir/ritonavir, $N(\%)$ & $26(43)$ \\
Low-molecular-weight heparin, $N(\%)$ & $20(34)$ \\
Survival & \\
Mortality rate, $\%$ & 34 \\
Mortality rate $<60$ years, $\%$ & 11 \\
Mortality rate $>60$ years, $\%$ & 45 \\
\hline
\end{tabular}

test capacity. This is also represented in the presenting symptoms: in the series by Lee et al. $61 \%$ had a fever, $47 \%$ a dry cough, $39 \%$ dyspnoea and $6 \%$ diarrhoea; these symptoms were all more frequently present in our series at presentation [3].

We did not observe any benefit of the given specific treatments for COVID-19. However, for the role of possible interventions in this category of patients, trials with larger, more uniform cohorts or randomised trials need to be conducted.

Overall, patients with a haematological disease seem to be more vulnerable to a more severe course of COVID-19 compared to patients without a malignancy, as already shown in the report by $\mathrm{He}$ et al. [1]. Pending a vaccine or treatment for COVID-19, precautions should be taken. Haematology departments should remain a COVID-19-free zone, patients and personnel should strictly comply with hygienic advices and social distancing, and patients and personnel should be tested even upon the mildest symptoms. Because of the expected long duration before normalisation of hospital care, treatment of the underlying disease should be continued when possible.

\section{Compliance with ethical standards}

Conflict of interest The authors declare that they have no conflict of interest. 
Publisher's note Springer Nature remains neutral with regard to jurisdictional claims in published maps and institutional affiliations.

\section{References}

1. He W, Chen L, Chen L, Yuan G, Fang Y, Chen W, et al. COVID-19 in persons with haematological cancers. Leukemia. 2020;34:1637-45.

2. Malard F, Genthon A, Brissot E, van de Wyngaert Z, Marjanovic Z, Ikhlef $S$, et al. COVID-19 outcomes in patients with hematologic disease. Bone Marrow Transplant. 2020. https://doi.org/10.1038/ s41409-020-0931-4.

3. Lee LYW, Cazier JB, Starkey T, Turnbull CD, UK Coronavirus Cancer Monitoring Project Team, Kerr R, et al. COVID-19 mortality in patients with cancer on chemotherapy or other anticancer treatments: a prospective cohort study. Lancet. 2020;395:1919-26.

4. Zhang L, Zhu F, Xie L, Wang C, Wang J, Chen R, et al. Clinical characteristics of COVID-19-infected cancer patients: a retrospective case study in three hospitals within Wuhan, China. Ann Oncol. 2020; 31:894-901. 